\title{
Plasma Levels of t-PA and PAI-1 Correlate With the Formation of Experimental Post-Surgical Peritoneal Adhesions
}

\author{
Clara Di Filippo, ${ }^{1,2}$ Alessandro Falsetto, ${ }^{3}$ Vito De Pascale, ${ }^{3}$ Elisabetta Tufariello, ${ }^{1}$ Domenico De Lucia, ${ }^{4}$ \\ Francesco Rossi, 1, 2 Michele D'Amico, 1, 2 and Antonio Cennamo ${ }^{3}$ \\ ${ }^{1}$ Section of Pharmacology L. Donatelli, Department of Experimental Medicine, Second University of Naples, 80138 Naples, Italy \\ ${ }^{2}$ Excellence Centre for Cardiovascular Diseases, Second University of Naples, 80138 Naples, Italy \\ ${ }^{3}$ Department of Anaesthetic Sciences and Emergency Surgery, Second University of Naples, 80138 Naples, Italy \\ ${ }^{4}$ Laboratory of Hemostasis and Thrombosis, Second University of Naples, 80138 Naples, Italy
}

Received 23 February 2006; Revised 20 April 2006; Accepted 20 April 2006

\begin{abstract}
This study has evaluated whether systemic changes of plasminogen activator (t-PA) and plasminogen activator inhibitor-1 (PAI-1) parallel the adhesions development and whether they could be used as predictors of adhesion risk. This has been studied in an animal model of post-surgical peritoneal adhesion by monitoring for 10 days the plasma and tissue levels of t-PA and PAI-1. The results showed that both tissular and plasmatic levels of t-PA were decreased in concomitance with the development of peritoneal adhesions. In contrast, PAI-1 was found increased into the tissue and into the plasma samples of the rats taken at 5 and 10 days time points. Inflammatory mediators such as ICAM-1, VCAM-1, and IL-6 within the peritoneal lavage fluid also correlated with the adhesion formation process. In conclusion, post-surgical peritoneal adhesions provide alterations of local inflammatory components and local and systemic fibrinolytic components, possibly with PAI-1 quenching t-PA. This may have potential for the identification of high-risk patients.
\end{abstract}

Copyright (c) 2006 Clara Di Filippo et al. This is an open access article distributed under the Creative Commons Attribution License, which permits unrestricted use, distribution, and reproduction in any medium, provided the original work is properly cited.

\section{INTRODUCTION}

Among the mechanisms and factors leading to the formation of post-surgical peritoneal adhesions, the fibrinolytic process and its mediators play a major role [1]. Fibrin is formed at injured sites either from bleeding or by post-traumatic inflammatory mechanisms. Fibrinous adhesions seem to be precursors to fibrous, and permanent adhesions [1]. Human recombinant tissue-type plasminogen activator (t-PA) was found to decrease adhesion formation [2-5] and postoperative inhibition of fibrinolysis to enhance the formation of adhesions [1]. Moreover, other studies suggest that the components of the plasmin system, particularly plasminogen activator inhibitor-1 (PAI-1), could be used as tissue markers of the propensity to develop adhesions after surgery and may have potential for identification of high-risk patients [6]. In other word, while t-PA is decreased during adhesions, PAI-1 is increased [6]. No study however was attempted to evaluate whether systemic changes of t-PA and PAI- 1 also parallel adhesions development, whether they correlate with local changes, and whether they could be used as predictors of adhesion risk. This has been studied by using an animal model of post-surgical peritoneal adhesion in rats. Moreover, the association between fibrinogenesis/fibrinolysis and inflammation has also been studied by the local measurements of inflammatory mediators such as intercellular adhesion molecule-1 (ICAM-1), vascular adhesion molecule1 (VCAM-1), and interleukin-6 (IL-6) within the peritoneal lavage fluid.

\section{MATERIALS AND METHODS}

Male Sprague-Dawley rats $(n=10)$ were marked with a pencil as 1 to 10 , anesthetized with urethane $(1.2 \mathrm{~g} / \mathrm{kg} \mathrm{ip})$, subjected to midline laparotomy, and had a blood sample $(500 \mu \mathrm{L})$ taken from the abdominal inferior vena cava. In the mean time, a sample of parietal peritoneal tissue was taken and an enterotomy was performed at the level of the ileum. The surgical incision was sutured with absorbable surgical wire $4 / 0$ in order to induce an inflammatory peritoneal insult. All of the surgical procedure was then ended by a nonabsorbable suture and the rats were placed in the recovering room for awakening. Five days after the surgery the rats were subjected to another laparotomy, had a new plasma and 
tissue sample taken, and were assayed for the development of peritoneal adhesions by means of qualitative and quantitative A score from 1 to 6 was established, and it was given as follows: 1 to the presence of poor and lapse adhesions in a limited peritoneal method. zone; 6 to the presence of dense adhesion and impossible access to peritoneal cavity. The same procedure was repeated after 10 days from the first surgery, having particular attention to keep always the same rat numbering over the time course considered. Each plasma and tissue sample was assayed for t-PA, PAI-1, F1 + 2, and von Willebrand. Tissue homogenate was prepared in Tris$\mathrm{HCl} 50 \mathrm{mM}$ (pH 7.2) containing $1 \mu \mathrm{M}$ leupeptin, $1 \mu \mathrm{M}$ pepstatin $\mathrm{A}$, and $200 \mu \mathrm{M}$ phenyl-methylsulfonylfluoride, and a Bradford assay was performed to assess total protein concentration.

Times 0,5 , and 10 days were also considered for the evaluation of local inflammatory mediators such as ICAM-1 and VCAM- 1 and the proinflammatory cytokine IL- 6 within the peritoneal lavage fluid. At each time point the peritoneal cavity was washed with $3 \mathrm{~mL}$ of PBS containing $3 \mathrm{mM}$ EDTA and $25 \mathrm{U} / \mathrm{mL}^{-1}$ heparin. Lavage fluid was then centrifuged at $400 \mathrm{~g}$ for $10 \mathrm{~min}$, and aliquots $(50 \mu \mathrm{L})$ supernatants were stored at -20 before biochemical determinations with commercially available Quantikine ELISA kits purchased from R\&D Systems (Oxfordshire, UK). The ELISAs showed negligible $(<1 \%)$ cross-reactivity with other cytokines (data furnished by the manufacturer).

\section{RESULTS}

The results showed that there is strict linear regression between the plasmatic levels of t-PA, PAI-1 and the tissue levels of these markers following the development of adhesions. Essentially, both tissular and plasmatic levels of t-PA were decreased $(-620 \pm 76 \%$ vs time 0$)$ after 5 days of intervention and continued to decrease within the next 5 days $(n=8)$ (Figure 1). The square coefficient of the regression analysis for the association between t-PA and adhesions was 0.955. In contrast, PAI-1 was increased in concomitance with the development with peritoneal adhesions (Figure 2). Particularly, there was an increase of $71 \pm 4 \%(P<.01$ vs time 0$)$ within the adhered tissues after 5 days, and an increase of the $83 \pm 6 \%$ $(P<.01$ vs time 0$)$ after 10 days from the surgery. Interestingly enough, this factor was also found increased into the plasma samples of the rats taken at 5 and 10 days time points (Figure 2). Regression analysis (square coefficient of 0.976) demonstrated a significant association between the score of the adhesions and the plasma levels of PAI-1. Overall, the mortality of the rats following the surgical insult and development of adhesions was $\sim 20 \%$. As shown by Figure 3, plasma concentration of $\mathrm{F} 1+2$ and von Willebrand factors were neither modified by the surgical procedure nor involved in adhesion formation.

Figure 4 shows the levels of ICAM-1, VCAM-1, and IL-6 in the peritoneal lavage fluid of rats following the induction of peritoneal surgical insult. Noteworthy, the levels of these mediators are significantly increased $(P<.01$ vs time 0$)$ after 5 and 10 days from the insult. The correlation with the

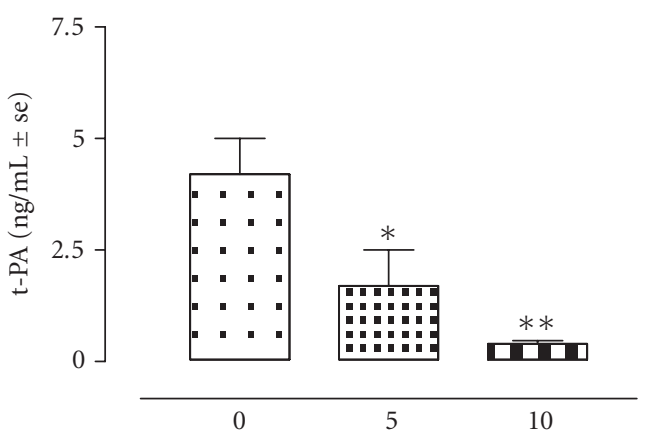

(a)

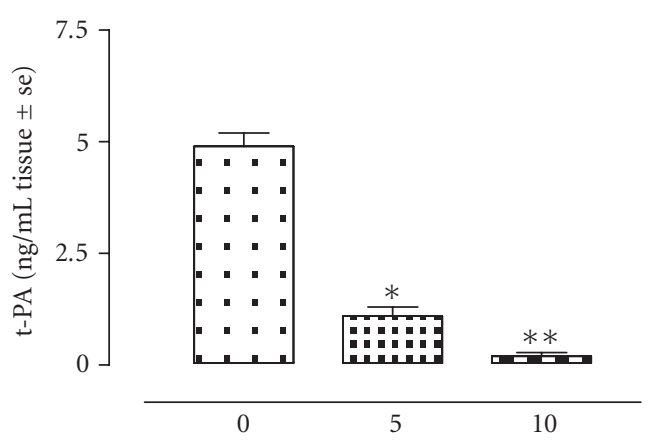

(b)

Figure 1: t-PA levels in rats $(n=8)$ subjected to experimental peritoneal adhesion development. The time is expressed in days. Significant differences from time 0 values are expressed as ${ }^{*} P<.01$; ${ }^{* *} P<.001$.

severity of the adhesion was determined by relative square coefficients of $0.954 ; 0.962 ; 0.879$ for ICAM-1, VCAM- 1 , and IL-6, respectively.

\section{DISCUSSION}

The formation of adhesions is the result of a balance between fibrinogenesis and fibrinolysis. The prevalence of one of these on the other produces the variability of the response to adhesions in patients. The present study, in agreement with others [6], showed that post-surgical peritoneal adhesions provide alterations of local fibrinolytic components, possibly with PAI-1 quenching t-PA. However, in general, each patient has a predisposition to form adhesions due to basal variability of the levels of some plasma and tissue markers. Our study raised interesting results on this point and argues concerns to follow in further studies, those of the possibility to predict adhesion formation and to understand the risk level for each patient to develop adhesions during preand post-operative periods with a simple plasma sample.

Plasma markers are related to those at tissue level and mirror their course in response to inflammatory stimuli during the time. t-PA and PAI seem to be very sensible of this phenomenon, even if with low specificity their involvement 


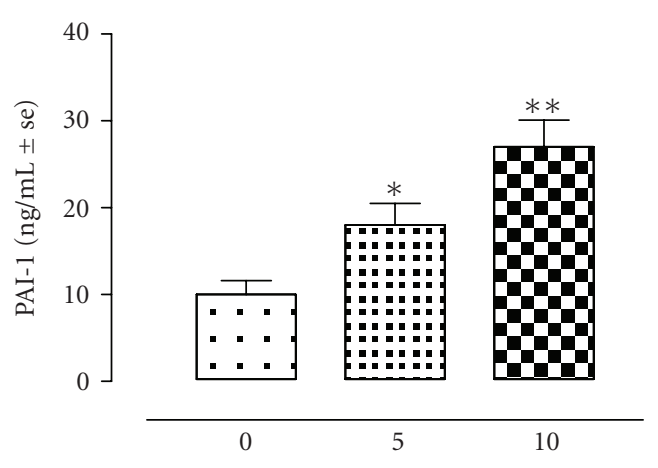

(a)

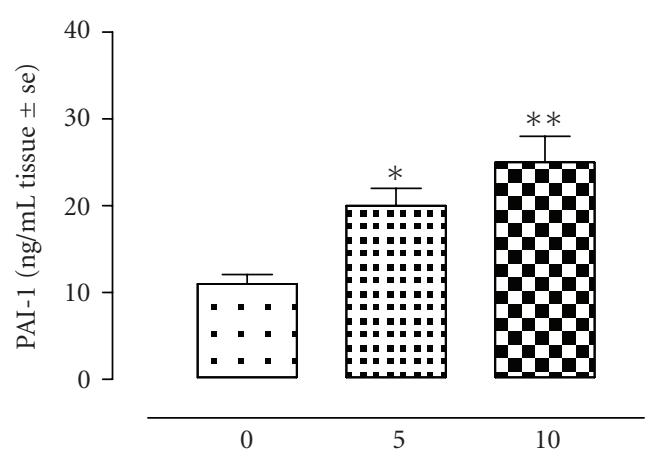

(b)

FIgURE 2: PAI-1 levels in rats $(n=8)$ subjected to experimental peritoneal adhesion development. The time is expressed in days. Significant differences from time 0 values are expressed as $* P<.05$; ** $P<.01$.

in fibrinolytic and fibrinogenic processes render these markers mirror of the events happening at tissue level. Indeed, the plasma values of both t-PA and PAI-1 measured during our experimental procedure parallel those measured within the adhered peritoneal tissue, evaluated at the same time points. Interestingly, time 0 shows that there is variability among the rats concerning the content of these factors especially at plasmatic compartment, some have high levels and some others low levels. This raises the question of whether they are directly correlated with the development of adhesion, that is, whether rats with low basal t-PA or high basal PAI-1 have more incidence or severity of adhesion while the contrary occurs with high basal t-PA and low PAI-1. A further study should discern this by a more appropriate protocol focused on the relation plasma levels of markers-time post-surgeryadhesion development.

An important component of the adhesion formation is the inflammatory response. During this phase leukocytes, cytokines, chemokines, and adhesion molecules play a key role [7]. ICAM-1 and VCAM-1 are the adhesion molecules expressed by mesothelial cells lining the peritoneal cavity, and IL- 6 is the most prominent cytokine expressed by the macrophages present within the peritoneum after the

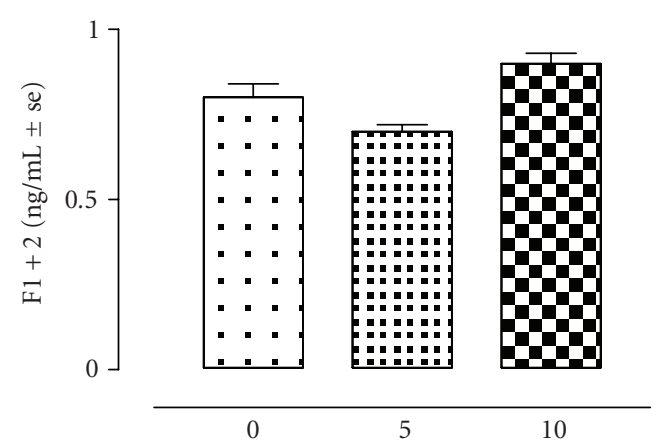

(a)

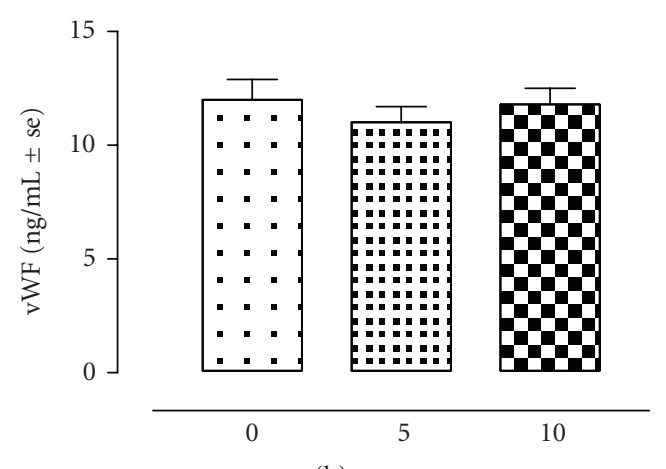

(b)

Figure 3: Plasma F1 +2 and von Willebrand levels in rats $(n=8)$ subjected to experimental peritoneal adhesion development. The time is expressed in days. Significant differences from time 0 values are expressed as ${ }^{*} P<.01 ;{ }^{* *} P<.001$.

adhesion formation [8]. The present study showed that the levels of these mediators within the peritoneal lavage fluid are very low at the moment of the induction of the surgical insult and rise during the 10 days of observation. In particular, the levels of ICAM-1, VCAM-1, and IL- 6 within the peritoneal lavage fluid seems to correlate with the severity of the adhesion formed. Animals showing mild adhesion also showed low levels of ICAM-1, VCAM-1, and IL-6; animals showing dense adhesion also showed high ICAM-1, VCAM-1, and IL6. Concerning the time course, these molecules are increased after 5 days with respect to time 0 and increased much more at 10 days time point. In contrast, the increase in IL- 6 levels observed at 5 days time point was not further changed 5 days later, in agreement with the concept that this cytokine is an adhesiogenic cytokine and its profile is indicative of wellestablished adhesion [8]. Also this cytokine is though to interact extensively with the fibrinolytic pathway [8].

In conclusion, post-surgical peritoneal adhesions provide alterations of local fibrinolytic components, possibly with PAI-1 quenching t-PA, but also they are mirrored by systemic changes of these two factors that may have potential for identification of high-risk patients. Adhesion formation also associates with increase of local inflammatory mediators. 


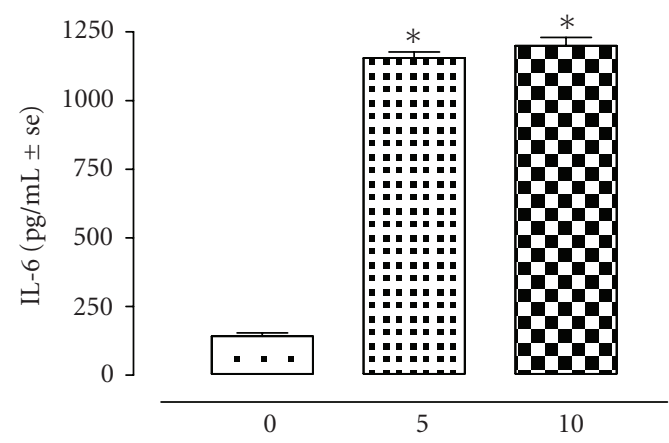

(a)

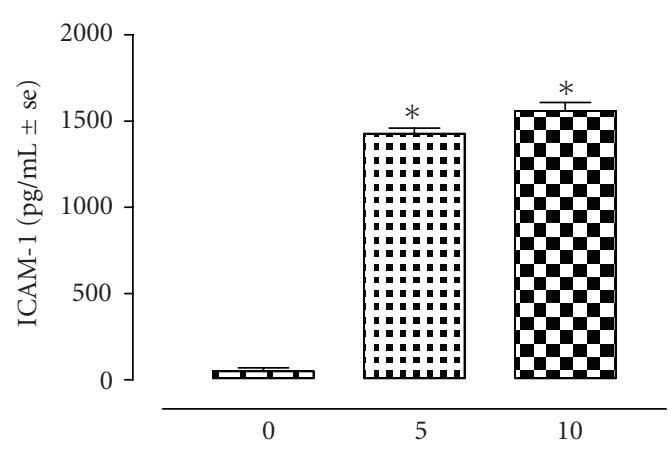

(b)

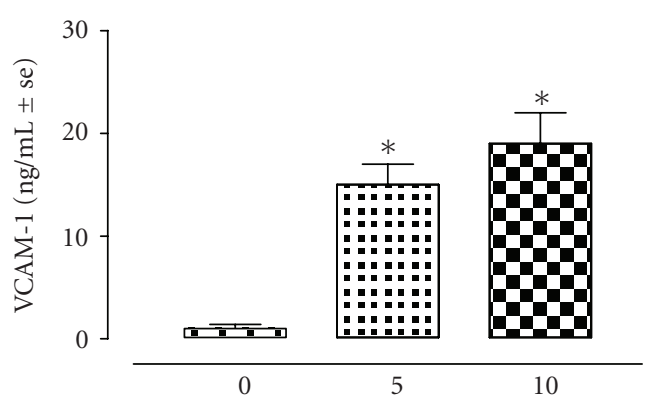

(c)

Figure 4: Levels of IL-6, VCAM-1, and ICAM-1 within the peritoneal lavage fluid of rats $(n=8)$ during the development of peritoneal adhesions. The time is expressed in days. Difference versus time 0 is represented as ${ }^{*} P<.001$.

\section{ACKNOWLEDGMENT}

This study was supported by MIUR, Rome, Italy.

\section{REFERENCES}

[1] Holmdahl L, Eriksson E, Al-Jabreen M, Risberg B. Fibrinolysis in human peritoneum during operation. Surgery. 1996;119(6):701-705.

[2] Doody KJ, Dunn RC, Buttram VC Jr. Recombinant tissue plasminogen activator reduces adhesion formation in a rabbit uterine horn model. Fertility and Sterility. 1989;51(3):509-512.

[3] Menzies D, Ellis H. Intra-abdominal adhesions and their prevention by topical tissue plasminogen activator. Journal of the Royal Society of Medicine. 1989;82(9):534-535.
[4] Dorr PJ, Vemer HM, Brommer EJP, Willemsen WNP, Veldhuizen RW, Rolland R. Prevention of postoperative adhesions by tissue-type plasminogen activator (t-PA) in the rabbit. European Journal of Obstetrics, Gynecology, and Reproductive Biology. 1990;37(3):287-291.

[5] Orita H, Fukasawa M, Girgis W, DiZerega GS. Inhibition of postsurgical adhesions in a standardized rabbit model: intraperitoneal treatment with tissue plasminogen activator. International Journal of Fertility. 1991;36(3):172-177.

[6] Ivarsson M-L, Bergström M, Eriksson E, Risberg B, Holmdahl L. Tissue markers as predictors of postoperative adhesions. British Journal of Surgery. 1998;85(11):1549-1554.

[7] Cheong YC, Shelton JB, Laird SM, et al. IL-1, IL-6 and TNF$\alpha$ concentrations in the peritoneal fluid of women with pelvic adhesions. Human Reproduction. 2002;17(1):69-75.

[8] Cheong YC, Laird SM, Li TC, Shelton JB, Ledger WL, Cooke ID. Peritoneal healing and adhesion formation/reformation. $\mathrm{Hu}$ man Reproduction Update. 2001;7(6):556-566. 


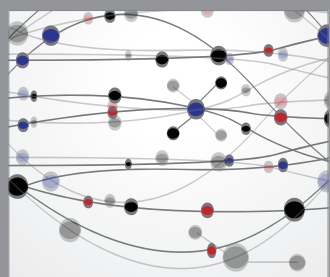

The Scientific World Journal
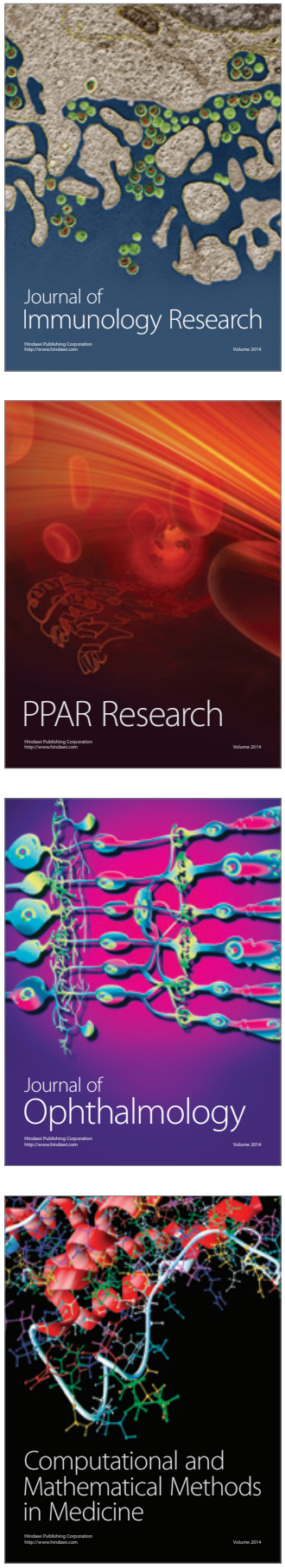

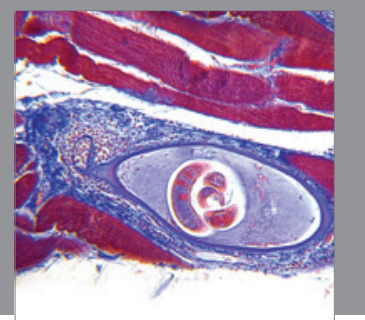

Gastroenterology

Research and Practice
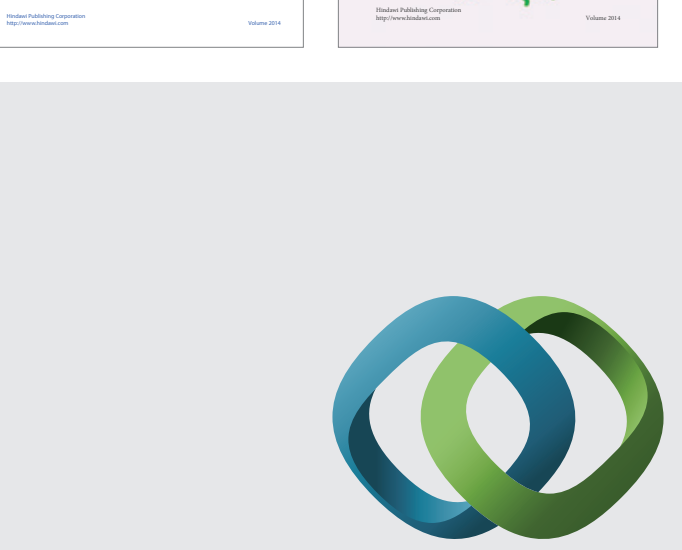

\section{Hindawi}

Submit your manuscripts at

http://www.hindawi.com
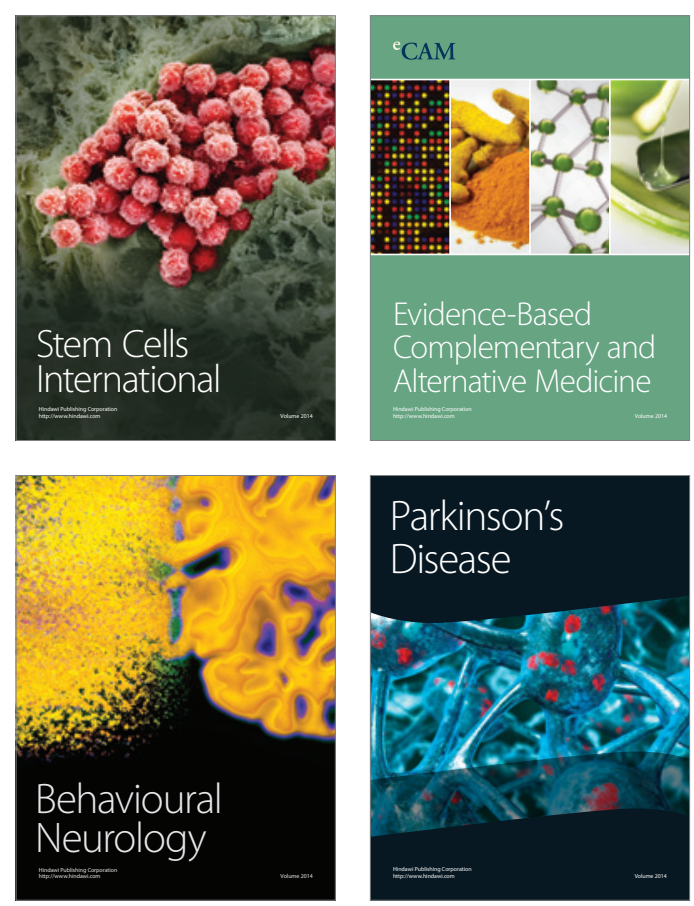

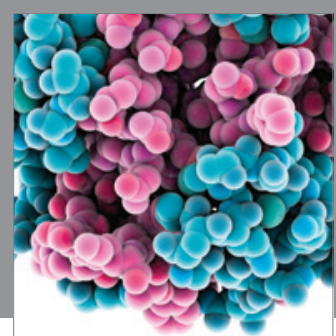

Journal of
Diabetes Research

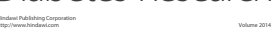

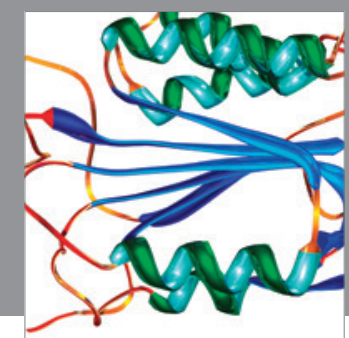

Disease Markers
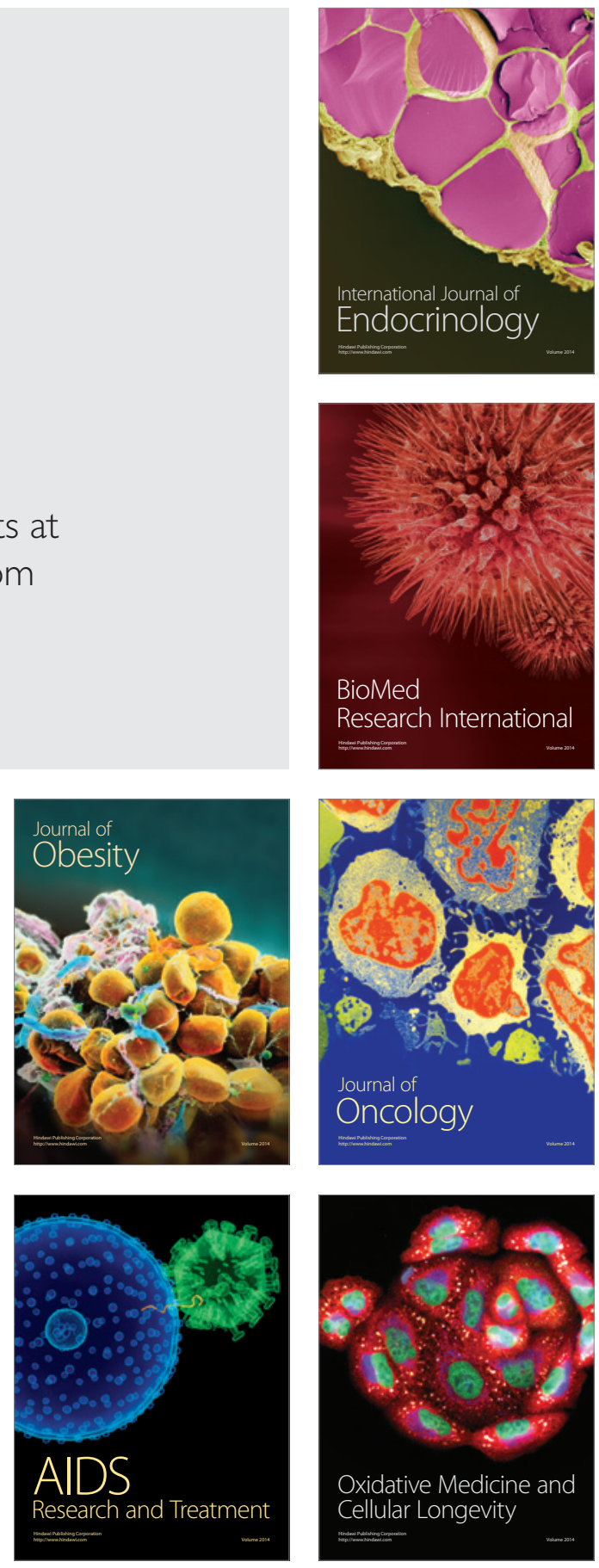